\title{
Failure Prediction in Commercial Banks with a Hybrid Prediction Model
}

\author{
Yinhua Li • Yong Shi • Anqiang Huang • \\ Haizhen Yang
}

Received: 3 May 2014 / Revised: 28 July 2014 / Accepted: 3 September 2014 /

Published online: 8 October 2014

(C) Springer-Verlag Berlin Heidelberg 2014

\begin{abstract}
In this paper, a new prediction model which was combined by trait recognition and SVM is examined through the use of accounting data measured 1 year prior to the commercial bank failure. To identity the features of problem banks and predict them as early as possible seems to be crucial for the stability of financial systems. Failure prediction models help to identify causes of failure, and thus lead to make a better understanding of bank operation. The contribution of six variable categories: profit, liquidity, loan risk, interest rate risk, capital and size are examined. The empirical data are collected from the Federal Deposit Insurance Corporation. Nine different comparing models, logistic regression method, C-SVM, Bayesian Network and so on were employed to justify the advantages of the hybrid method. In addition, the new method outperformed nine prediction models in overall accuracy. It was also shown
\end{abstract}

\footnotetext{
Y. Li (凶)

Post Doctoral Scientific Research Workstation, China Merchants Bank, Shenzhen 518000, China e-mail: yinhua_li@cmbchina.com

Y. Shi $\cdot$ H. Yang

Research Center on Fictitious Economy and Data Science, Chinese Academy of Sciences, Beijing 100190, China

e-mail: yshi@gucas.ac.cn

H. Yang

e-mail: haizheny@gucas.ac.cn

Y. Shi

College of Information Science and Technology, University of Nebraska at Omaha, Omaha, NE 68118, USA
}

A. Huang

School of Economics and Management, Beihang University, Beijing 100191, China

e-mail: anqianghuang@sem.buaa.edu.cn 
that ROA, liquidity assets and short-term gaps are sound predictors for bank failure prediction.

Keywords Early warning $\cdot$ Commercial bank · Failure $\cdot$ Hybrid $\cdot$ Trait recognition · SVMs

\section{Introduction}

August 2007, Bear Stearns, which is the top fifth investment bank in America, announced that two funds under its supervision went insolvent. This signified that the serious subprime crisis had spread in America and wrapped around the globe rapidly. A bust housing market in the U.S. led to massive losses on mortgage-related securities, which were magnified by massive amounts of leverage in derivatives tied to those securities. Commercial banks and other financial institutes were subjected to loan losses due to the rupture of fund chain. When a bank is unable to meet its obligations to depositors and others, it will go into bankruptcy.

As a result, these losses have forced regulators to seize a growing number of banks and other financial institutions in America and other countries around the world, leading to a freeze-up in credit markets and a global recession. Therefore, it becomes critically important to forecast bank failure for policy makers and bank risk managers. In order to forecast bank failure precisely, it is necessary to grasp the most determining factors which lead to bank failure and establish effective models to predict bank failure.

The elements which lead to bank failure are sophisticated. Generally speaking, they include external factors and internal operation problems. Economic recessions have negative effects on bank systems. However, the direct reason for bank failures depends on their operation condition.

Existing research demonstrate that historical data from accounting sheets have the ability to predict future operating condition of banks [1,2]. It is far-reaching to establish an effective early warning model for commercial banks. Firstly, it is propitious to allocate resources for central banks. The timely detection of troubled banks can help reduce the cost of regulation and supervision. Secondly, it can make full use of historical data. The attributes variables mainly come from the accounting sheets of the banks, based on which bank failure can be forecasted. Thirdly, it can provide an evaluation standard to supervisory board to judge whether banks are given the proper rating.

With the development of computer technology, intelligent models are widely applied in the area of bank failure prediction. This paper has two problems to deal with. One is to examine if the performance of the trait recognition method is efficient in different data sets. According to the test result, a new method is proposed to predict commercial bank failure. Another one is to identify what the crucial factors are under the new background.

The main contributions of this paper are twofold. Firstly, we propose a hybrid method and compare its result with nine algorithms using accounting data of U.S. commercial banks under the subprime mortgage crisis background. Secondly, try to analyze and select the most important predictors using F-Score algorithm. 
The remaining of the paper is organized as follows. The next section reviews current literature on how to establish the early warning system for bank failure prediction. In the third section, we will introduce the whole construction process of hybrid method. After that, in the forth section, empirical study and results will be presented. In the last section, the conclusions are presented.

\section{Literature Review on Risk Supervision and Prediction for Banks}

In the research field of bank risk supervision, there are large number of papers focus on credit risk, market risk or operational risk [3-13] Due to a new round of economy deteriorating, especially, bank failure prediction has become research focus again $[1,3]$. Two aspects of questions are involved in bank failure prediction. One is how to select proper predictors. The other is which algorithms should be utilized. This review is followed by an introduction of the two questions.

In many applications of risk prediction, data are commonly presented as highdimensional feature vectors. Therefore, the selection of predict factors is useful and have crucial impact on the results of prediction. The methods of feature selection can be categorized into three groups. Filter methods are independent of the classifiers [14]. Wrapper methods evaluate their fitness based on classification accuracy [15]. And embedded method incorporate feature selection in the classifier objective function $[16,17]$.

The use of accounting information for failure prediction has a long history because of its public availability [19]. Jin [1] identified low capital and risky assets as major reasons for bank failure. Then, the accounting variables like loan loss provisioning, balance sheet strength, and loan quality and mix are chosen as proxy of important factors. Brunnermeier [20] has concluded that the rise in popularity of securitized products ultimately led to a flood of cheap credit quality.The cheap credit and loan lending standard resulted in the housing frenzy that laid the foundations for the crisis. Lanine [19] considered that bank failures are mainly caused by deteriorating liquidity of the banks, but deteriorating profit and low levels of capital adequacy are also important predictors.

In the light of second question, studies have proven that data mining is effectively used for knowledge discovery and decision making [21]. Data mining techniques are also broadly used for prediction of financial distress recently [22]. The most hotly used techniques recently in financial distress prediction are support vector machines (SVMs), proposed by Vapnik and his cooperators in 1990s [23], and firstly applied into the area of financial distress prediction after 2005. In addition, some another methods are commonly used, including artificial neural network [24,25], decision tree [26], AHP-based model [27], trait recognition [19,28], and hybrid models [29]. Some intelligent models do not have explanation of the results like artificial neural network, decision tree. This character limits to support decision making.

A non-parametric method named trait recognition was firstly introduced into the research of bank failure prediction by Kolari [28]. Ref. [28] sampled 1950 American commercial banks during 1984 and 1985. The accuracy in both original split sample and holdout split sample is more than $95 \%$. The relative work [19] on trait recognition 
investigated the 582 failed banks dating from 1988 to 2004 in Russia. The accuracy of their modified trait recognition model is $85.1 \%$ in holdout split sample. The main conclusions are summarized in Table 1.

Although the trait recognition technique has some advantages, it also has some deficiencies. The process of selecting good or bad feature is arbitrary, which will lead to missing the important information during the selection process. Besides, the robustness of the trait recognition model need further testing in different period data samples. The linear SVM may overcome the shortcoming of the trait recognition. It is able to give a specific weight to every input variable. Therefore, the contribution of each input variable is clear. Meanwhile the encoding technique in trait recognition takes the correlation between two or three variables into consideration. In this paper, a hybrid model of trait recognition and liner kernel SVM is proposed to overcome their shortcomings.

\section{Hybrid of Trait Recognition (TR) and Support Vector Machines (SVMs)}

To overcome the deficiencies in existing trait recognition models, we combine information encoding technique that is used in trait recognition with the SVMs model reasonably. For more details on TR, you can refer to the literature by Kolari et al. [28].The motivation to establish a hybrid prediction model is to combine the advantages of both TR and SVMs. The advantages of the TR technique are that it imposes no priori assumptions on the variables. TR emphasizes the interactions among the independent variables and can give useful knowledge on attribute union.

In the next section, we will introduce the whole process of constructing the new hybrid prediction model, see Fig. 1. First of all, try to select the proper accounting factors as comprehensive as possible. The liquidity of the bank is usually used to measure the bank health.

Under the new era of global financial crises, liquidity of the bank and quality of assets should be examined by quantitative models, including whether or not they have anticipative correlation with commercial bank failure. The accounting factors we chose contain 12 attributes which belong to six categories. We proxy bank profit capability with the following variables: profit margin, ROA, ROE. The loan quality particularly loan loss provision is crucial in determining the health of a bank [1]. In addition, we choose two additional attributes to proxy loan risk: loan exposure and loan funding. The liquidity is also deemed as one of most important accounting attributes of bank. In this category, we choose liquidity asset and cash asset ratio. Further, this study considers capital, interest rate risk and size to portray the condition of the commercial bank from various angles, see Table 2.

\subsection{Step1. Attribute Selection and Quantitative Transformation}

The first step is to construct a binary string according to financial ratios of each bank. Based on sample data, the distribution of these ratios can be plotted. Then for each ratio, two breakpoints are established to segment the distribution of observation into three segments: (1) High segment purely consisting of non-failed banks is denoted 


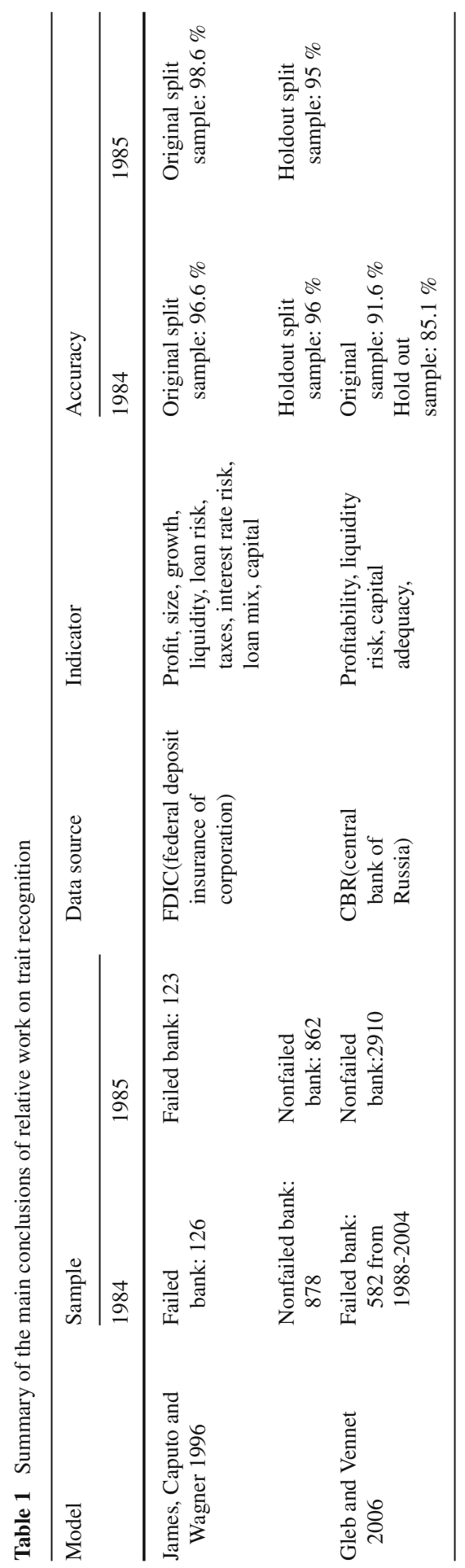




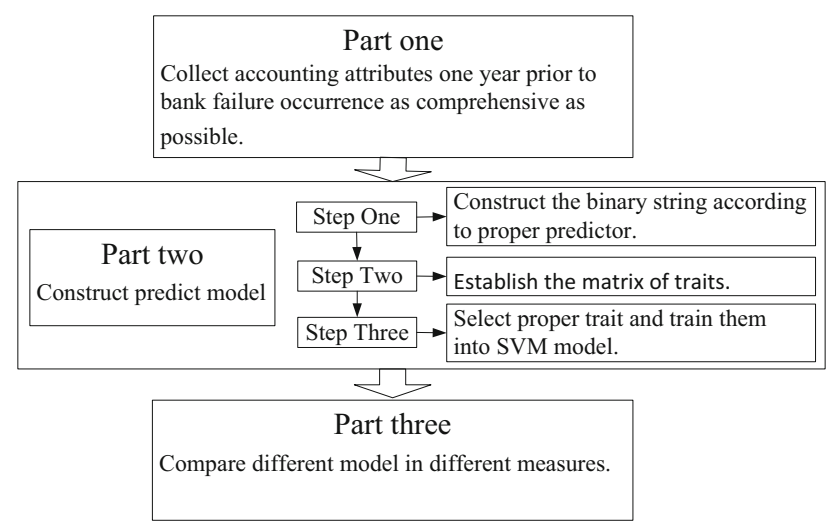

Fig. 1 The whole process of the hybrid prediction method

Table 2 Accounting ratio for each commercial bank

\begin{tabular}{llll}
\hline Category & Accounting ratio & Remarks & Abbreviation \\
\hline Profit & Profit margin & Net interest income/Total assets & PM \\
& ROA & Net income after taxes/Total assets & ROA \\
& ROE & Net income after taxes/Total equity & ROE \\
Liquidity & Liquid asst & Total securities/Total assets & LA \\
& Cash asset ratio & Cash and due & CAR \\
& & from depository & \\
Loan risk & & institutions & LE \\
& Loan exposure & Net loans and leases/Total assets & LF \\
& Loan funding & Net loans and leases/Total deposits & PR \\
Capital & Provision rate & Provision for possible loan losses/Total assets & CG \\
& Capital growth & (Total equity(t)-Total equity(t-1))/Total equity & CR \\
Interest rate risk & Capital ratio & Total equity/Total assets & STG \\
Short-term gap & (Assets adjustable 1 day - 1 year - liabilities & adjustable1 day to 1 year)/ Total assets & ASS \\
\hline
\end{tabular}

as "H" and coded as " 00 "; (2) The mixture of failed and non-failed banks is denoted as "M" and coded as "01"; (3) Low segment purely consisting of failed banks is denoted as "L" and coded as " 11 ". Generally, the observation M is described by the binary code $M_{1} M_{2} \ldots M_{l}, l$ is the length of the string. When the cutoff points are chosen, we allow $10 \%$ failed banks existing among the non-failed banks in the high section. In a similar way, $10 \%$ non-failed banks are allowed to exist among the failed banks in the low section. However, either higher or lower value of some attributes represents the failed banks or healthy bank. Therefore, we dropped these attributes. 
Table 3 The matrix of traits for a bank

\begin{tabular}{rrrrrrrr}
\hline $\mathrm{p}$ & $\mathrm{q}$ & $\mathrm{r}$ & $\mathrm{PQR}$ & $\mathrm{p}$ & $\mathrm{q}$ & $\mathrm{r}$ & $\mathrm{PQR}$ \\
\hline 1 & 1 & 1 & 000 & 2 & 3 & 3 & 000 \\
2 & 2 & 2 & 000 & 2 & 4 & 4 & 011 \\
3 & 3 & 3 & 000 & 3 & 4 & 4 & 001 \\
4 & 4 & 4 & 111 & 1 & 2 & 3 & 000 \\
1 & 2 & 2 & 000 & 1 & 2 & 4 & 001 \\
1 & 3 & 3 & 000 & 1 & 3 & 4 & 001 \\
1 & 4 & 4 & 011 & 2 & 3 & 4 & 001 \\
\hline
\end{tabular}

Table 4 Top ten scoring attributes combination

\begin{tabular}{llcl}
\hline Rank & Attributes combination & Rank & Attributes combination \\
\hline 1 & ROA and CG & 6 & ROA and STG \\
2 & ROA and LA and CG & 7 & ROA and LE and CG \\
3 & ROA and LA & 8 & ROE and PM and CG \\
4 & ROA and CG and STG & 9 & ROA and CG and CR \\
5 & ROA and LA and STG & 10 & ROA and LA and LE \\
\hline
\end{tabular}

\subsection{Step2. Establish the Matrix of Traits}

To extract the distinctive feature that represent the common pattern of different groups of observations, the string of binary code should be transferred into a matrix of trait. Each trait is comprised of an array with six integers: $T=p, q, r, P, Q, R$. The symbols $\mathrm{p}, \mathrm{q}, \mathrm{r}$ represents the positions in the binary string from left to right. $\mathrm{P}, \mathrm{Q}, \mathrm{R}$ give the values of the binary code at the position $\mathrm{p}, \mathrm{q}$ and $\mathrm{r}$. Take the binary string " 0001 " for example; the matrix of traits for this bank is as follows (Tables 3, 4):

\subsection{Step3. Select Proper Trait and Train them into SVM Model}

After carrying out step1 and step2 described above, a series of trait which reflect the operating conditions 1 year prior to bank failure are received. However, not all traits are useful to predict the bank failure. Some of them may contain repetitive or noisy information. Therefore, we need to select features from large amounts of traits. By utilizing F-Score [14], it can give an explicit score to each attribute and rank them in descending order. The larger the attribute score is, the better the prediction outcome is.

The support vector machine proposed by Vapnik [23] is one of the most popular algorithms in machine learning research. The main idea of this algorithm is that it maps the input vector $\mathrm{x}$ into a high-dimensional feature space $\mathrm{Z}$ through some nonlinear mapping, chosen a priori. 
Given a training set $T=\left\{\left(x_{1}, y_{1}\right), \ldots,\left(x_{l}, y_{l}\right)\right\} \in\left(R^{n} \times \gamma\right)^{l}, x_{i} \in R^{n}, y_{i} \in \gamma=$ $(1,-1), i=1, \ldots, l$; Select proper penalty coefficient $\mathrm{C}, \mathrm{C}>0$. A convex quadratic programming problem is constructed as function (3.1).

$$
\begin{array}{ll}
\min _{\alpha} & \frac{1}{2} \sum_{i=1}^{l} \sum_{j=1}^{l} y_{i} y_{j}\left(x_{i} \cdot x_{j}\right) \alpha_{i} \alpha_{j}-\sum_{j}^{l} \alpha_{j} \\
\text { s.t. } & \sum_{i=1}^{l} y_{i} \alpha_{i}=0 \\
& 0 \leq \alpha_{i} \leq C, i=1, \ldots, l
\end{array}
$$

The parameter $\alpha^{*}=\left(\alpha_{1}^{*}, \alpha_{2}^{*} \ldots \alpha_{l}^{*}\right)^{T}$ can be solved. Then calculate the $b^{*}$ as function (3.2),

$$
b^{*}=y_{i}-\sum_{i=1}^{l} y_{i} \alpha_{i}^{*}\left(x_{i} \cdot y_{j}\right)
$$

Finally, construct the optimal separating hyper plane $\left(\omega^{*} \cdot x\right)+b^{*}=0$, and the decision function $f(x)$ can be presented as function (3.3).

$$
f(x)=\operatorname{sgn}(g(x))
$$

where

$$
g(x)=\sum_{i=1}^{l} y_{i} \alpha_{i}^{*}\left(x_{i} \cdot x\right)+b^{*}
$$

The new proposed method has three advantages. Firstly, it will not miss any useful information. Secondly, it can give a clear result whether a bank belongs to the failed group or the non-failed group. And third, it can consider the correlations among two or three attributes during the encoding process.

In the third part, we will employ nine different algorithms to examine the effect of hybrid method using four model performance measures respectively.

\section{Experiment and Results}

The data is collected from the Federal Deposit Insurance Corporation (FDIC), including both failed and non-failed commercial banks. The total number of failed bank is 158. And we select the same number of non-failed bank corresponding to the failed bank. Every bank is described by eight financial ratios, including ROA, ROE, Profit margin (PM), Liquidity assets (LA), Loan exposure (LE), Capital growth (CG), Short term gap (STG), Capital ratio (CR). These financial ratios cover six aspects of financial conditions, including profit, liquidity, growth, loan risk, interest rate risk and capital.

For each bank in training sample, there are 816 traits reflecting the information among any two or three financial ratios. We utilize F-Score method to give a score to each attribute. The top ten highest score attributes or attributes union are listed in Table 5. In the top ten scoring attributes union, ROA is the most important accounting 
Table 5 Result comparison between different algorithms

\begin{tabular}{llrlrrrrr}
\hline Algorithm & $\begin{array}{l}\text { Overall } \\
\text { accuracy }\end{array}$ & Rank & precision & Rank & $\begin{array}{l}\text { True pos- } \\
\text { itive rate }\end{array}$ & $\begin{array}{l}\text { Rank } \\
\text { negative } \\
\text { rate }\end{array}$ & $\begin{array}{l}\text { True } \\
\text { Rank }\end{array}$ \\
\hline Bayesian network & 0.9177 & 4 & 0.9240 & 7 & 0.9125 & 7 & 0.9230 & 6 \\
Naïve Bayes & 0.9240 & 2 & 0.9303 & 5 & 0.9187 & 5 & 0.9294 & 4 \\
RBFNetwork & 0.8892 & 8 & 0.9493 & 2 & 0.8474 & 10 & 0.9424 & 3 \\
J48 & 0.8987 & 6 & 0.9620 & 1 & 0.8539 & 9 & 0.9565 & 1 \\
ADTree & 0.8829 & 9 & 0.7658 & 9 & 1.0000 & 1 & 0.8102 & 9 \\
LADTree & 0.8797 & 10 & 0.7594 & 10 & 1.0000 & 1 & 0.8061 & 10 \\
NBTree & 0.9240 & 3 & 0.9303 & 6 & 0.9187 & 6 & 0.9294 & 5 \\
Logistic & 0.8984 & 7 & 0.8747 & 8 & 0.9191 & 4 & 0.8688 & 8 \\
C-SVM & 0.9177 & 5 & 0.9492 & 3 & 0.8984 & 8 & 0.9463 & 2 \\
Hybrid of TR and SVM & 0.9385 & 1 & 0.9430 & 4 & 0.9427 & 3 & 0.9146 & 7 \\
\hline
\end{tabular}

attribute which represents profit ability of commercial bank. Further, ROA combined with CG, LA and STG are better predictors then other attributes union.

In order to verify the predict power of the hybrid model further, we compare its performance with nine other prediction models. They are Bayesian Network, Naïve Bayes, RBF Network, J48, ADTree, LADTree, NBTree, logistic model and SVMs. The input attributes are accounting variables selected in Sect. 3 the first part. Moreover, the result for each algorithm is based on ten-fold cross validation.

Accuracy and error rate are important measures of classification algorithms in financial risk prediction [30]. This paper utilizes overall accuracy, precision, true positive rate and true negative rate, see function (4.1)-(4.4). Accuracy measures the overall correctly classified instance.

$$
\text { Overall accuracy }=\frac{T N+T P}{T N+F P+T P+F N}
$$

where TP, TN, FP, and FN represent true positive, true negative, false positive, and false negative, respectively.

Precision is the number of classified positive or failed instances that actually are positive instances.

$$
\text { Precision }=\frac{T P}{T P+F P}
$$

True positive rate is the number of correctly classified positive or failed instances. TP rate measures how well a classifier can recognize failed instance. It is also called sensitivity.

$$
\text { True positive rate/sensitivity }=\frac{T P}{T P+F N}
$$

True negative rate is the number of correctly classified negative or normal instances. $\mathrm{TN}$ rate measures how well a classifier can recognize normal instance. It is also called specificity. 


$$
\text { True negative rate } / \text { specificity }=\frac{T N}{T N+F P}
$$

From the ten-fold cross validation results, the hybrid method ranks the first in overall accuracy, the third in precision and TP rate and the fourth in Precision.

\section{Conclusions and the Future Work}

The hybrid method is an important one to predict commercial bank failure. It can make full use of information represented by financial data. The empirical results conclude that it outperforms the other nine algorithms in overall accuracy by ten-fold cross validation.

Among the traits, ROA is the most important accounting attribute which represents profit ability of commercial bank. Further, ROA combined with CG, LA and STG are batter predictors then other attribute combination.

The hybrid method proposed in this paper still has some deficiencies. For example, the result of the method has a strong relationship with the training sample and the selection of cutoff point is arbitrary to some degree, which are worthy of further research.

Acknowledgments This research was partially supported by a grant from National Natural Science Foundation of China (\#70621001,\#10601064,\#71071148,\#70921061,\#71110107026,\#70933003). Part of this paper was presented at International Conference on Pacific Basin Finance, Economics, Accounting, and Management (PBFEAM2011). The authors thank their colleagues William A. Jefferson and Yibing Chen for helping with polishing the languages.

\section{References}

1. Jin JY, Kanagaretnama K, Lobo GJ (2011) Ability of accounting and audit quality variables to predict bank failure during the financial crisis. J Bank Financ 35(11):2811-2819

2. Kaminsky G, Reinhart G (1999) The twin crises: the cause of banking and balance-of-payments problems. Am Econ Rev 89(3):473-500

3. Better M, Glover F, Kochenberger G, Wang H (2008) Simulation optimization: applications in risk management. Int J Inf Technol Decis Mak 7(4):571-587

4. Shi Y, Peng Y, Kou G, Chen Z (2005) Classifying credit card accounts for business intelligence and decision making: a multiple-criteria quadratic programming approach. Int J Inf Technol Decis Mak 4:581-600

5. Shi Y, Tian YJ, Kou G, Peng Y, Li JP (2011) Optimization based data mining: theory and applications. Springer, Berlin

6. Shi Y, Peng Y, Xu W, Tang X (2002) Data mining via multiple criteria linear programming: applications in credit card portfolio management. Int J Inf Technol Decis Mak 1(1):131-151

7. Peng Y, Kou G, Shi Y, Chen Z (2008) A multi-criteria convex quadratic programming model for credit data analysis. Decis Support Syst 44(4):1016-1030

8. Li J, Sun X, He W, Tang L, Xu W (2009) Modeling dynamic correlations and spillover effects of country risk: evidence from Russia and Kazakhstan. Int J Inf Technol Decis Mak 8(4):803-818

9. Li J et al (2010) An evolution-strategy based multiple kernels multi-criteria programming approach: the case of credit decision making. Int J Inf Technol Decis Mak 51(2):292-298

10. Li J, Feng J, Chen J (2009) A piecewise-defined severity distribution-based loss distribution approach to estimate operational risk: evidence from Chinese National Commercial Banks. Int J Inf Technol Decis Mak 8(4):727-747

11. Böcker K, Klüppelberg C (2010) Multivariate models for operational risk. Quant Financ 10(8):855-869 
12. Li J, Feng J, Chen J (2009) A piecewise-defined severity distribution based loss distribution approach to estimate operational risk: evidence from Chinese National Commercial Banks. Int J Inf Technol Decis Mak 8(4):727-747

13. Kou G, Peng Y, Shi Y, Wise M, Xu W (2005) Discovering credit cardholders' behavior by multiple criteria linear programming. Ann Oper Res 135(1):261-274

14. Chen YW, Lin CJ (2006) Combining SVMs with various feature selection strategies. Feature extraction, foundations and applications. Springer, New York

15. Inza I et al (2004) Filter versus wrapper gene selection approaches in DNA microarray domains. Artif Intell Med 31(2):91-103

16. Li J et al (2007) Feature selection via least squares support feature machine. Int J Inf Technol Decis Mak 6(4):671-686

17. Weston J et al (2003) Use of the zero-norm with linear models and kernel methods. J Mach Learn Res 3:1439-1461

18. Adrian T, Shin HS (2010) Liquidity and leverage. J Financ Intermed 19(3):418-437

19. Lanine G, Vennet RV (2006) Failure prediction in the Russian bank sector with logit and trait recognition models. Expert Syst Appl 30(3):463-478

20. Brunnermeier MK (2009) Deciphering the liquidity and credit crunch 2007-2008. J Econ Perspect 23(1):77-100

21. Peng Y et al (2008) A descriptive framework for the field of data mining and knowledge discovery. Int J Inf Technol Decis Mak 7(4):639-682

22. Kumar PR, Ravi V (2007) Bankruptcy prediction in banks and firms via statistical and intelligent techniques-a review. Eur J Oper Res 180(1):1-28

23. Vapnik VN (1995) The nature of statistical learning theory. Springer, Berlin

24. Gelik AE, Kararepe Y (2007) Evaluating and forecasting banking crises through neural network models: an application for Turkish banking sector. Expert Syst Appl 33(4):809-815

25. Huang W et al (2004) Forecasting foreign exchange rates with artificial neural networks: a review. Int J Inf Technol Decis Mak 3(1):145-165

26. Marais ML, Wolfson Patel JM (1984) The experimental design of classification models: an application of recursive partitioning and bootstrapping to commercial bank loan classification. J Account Res 22:87-113

27. Peng Y et al (2011) Ensemble of software defect predictors: an AHP-based evaluation method. Int J Inf Technol Decis Mak 10(1):187-206

28. Kolari J, Michele C, Drew W (1996) Trait recognition: an alternative approach to early warning systems in commercial banking. J Bus Financ Account 23(9):1415-1434

29. Li J et al (2011) Country risk forecasting for major oil-exporting countries: a decomposition hybrid approach. Submitted to Computers \& Industrial Engineering. doi:10.1016/j.cie.2011.12.003

30. Peng Y, Gang Kou G, Shi Y (2011) An empirical study of classification algorithm evaluation for financial risk prediction. Appl Soft Comput 11:2906-2915

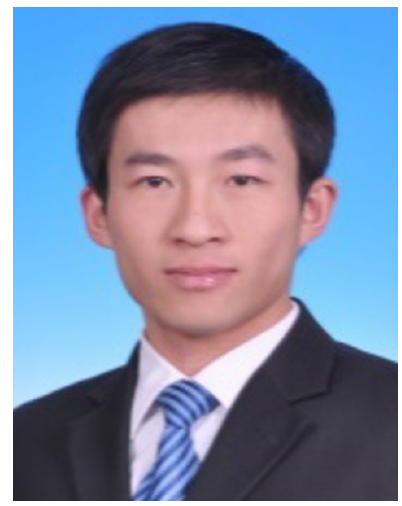

Yinhua Li received the Ph.D. degree in Management Science and Engineering from University of Chinese Academy of Sciences in 2013. He is currently a Post-Doctor of China Merchants Bank, Shenzhen, China. His research interests include financial risk prediction, text mining and machine learning. He has published nine papers about settlement risk prediction, customer behaviors analysis, international capital flow, etc. 

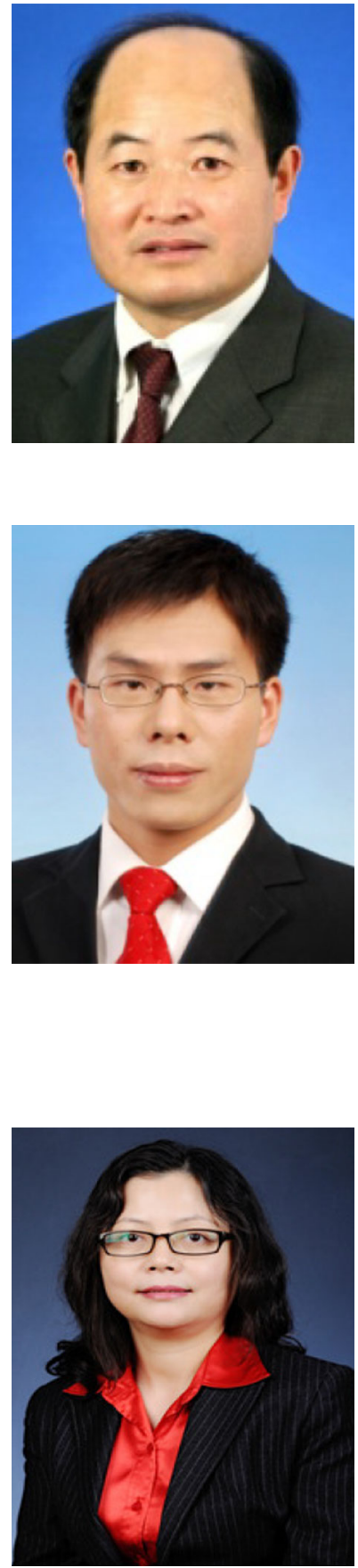

Yong Shi serves as the Executive Deputy Director, Chinese Academy of Sciences Research Center on Fictitious Economy \& Data Science and Director, the Key Research Lab on Big Data Mining and Knowledge Management, Chinese Academy of Sciences. He has been the Union Pacific Chair of Information Science and Technology, University of Nebraska at Omaha, USA. Dr. Shi's research interests include business intelligence, data mining, and multiple criteria decision making. He has published more than 22 books, over 200 papers in various journals and numerous conferences/proceedings papers.

Anqiang Huang received his Master degree in Management Science and Engineering from University of Chinese Academy of Sciences in 2010, and the Ph.D. degree in Management Science and Engineering in Beihang University in 2014. He has published eight papers on container throughput forecasting during his doctorial phase. He is currently working in School of Economics and Management, Beijing Jiaotong University. His research interests include forecasting theory and application, intelligent knowledge management, logistics and supply chain management, etc.

Haizhen Yang received a Master degree and Ph.D. degree in Management School from Xian Jiaotong University in 1990 and 2000. Currently she is a professor of School of Management in the University of Chinese Academy of Sciences. She also is a adjunct professor of Research Center on Fictitious Economy and Data Science, Chinese Academy of Sciences. Her current research fields include international capital movement and financial stability, economic analysis and forecasting, banking crises identification and early warning, investment analysis and portfolio management. 